\title{
Thrombocytopenia - An Indicator of Impending Complications in Dengue Virus Infection
}

\author{
Kanthishree B. Haritsa ${ }^{1}$, S. Sangeetha ${ }^{1} *$ and Mamatha B. Patil ${ }^{2}$ \\ ${ }^{1}$ Department of Microbiology, ${ }^{2}$ Department of General Medicine, Rajarajeswari Medical College \\ and Hospital, Bangalore, Karnataka, India \\ *Corresponding author
}

\section{A B S T R A C T}

Dengue virus infection is a major public health problem in urban, semi-urban and rural India. At times, the platelet count is the only accessory laboratory test available in the

Keywords

Thrombocytopenia, DHF, DSS, ELISA, Peripheral Health System.

Article Info

Accepted:

04 November 2017

Available Online:

10 October 2017 peripheral areas. Thrombocytopenia in clinically suspected patients of dengue infection can serve as a predictor of DHF or DSS and guide in the institution of early supportive and maintenance therapy. Hence in this study, correlation of platelet count with dengue parameters was done keeping in mind the constraints in the facilities in the peripheral health system. The study was conducted in a tertiary care teaching hospital. A total of 86 serum samples were subjected to NS1 ELISA and IgM ELISA. Platelet count of all the patients was noted. Chi square test was applied for analysis of categorical data. Out of 86 clinically suspected cases, 64 patients were positive for dengue parameters and remaining 22 patients were negative. There was good association between low platelet count and dengue positive parameters than with dengue negative parameters. ( $\mathrm{P}$ value $<0.001)$ In clinically suspected cases of dengue infection, if thrombocytopenia is observed, early and prompt therapy should be instituted in order to reduce the morbidity and mortality due to complications of dengue infection.

\section{Introduction}

Dengue virus infection is a major public health problem in India. Dengue viruses can cause a wide variety of clinical illnesses ranging from mildly symptomatic dengue fever (DF) to more dangerous clinical conditions with capillary leakage syndrome such as dengue shock syndrome (DSS) and dengue hemorrhagic fever (DHF) (Gupta et al., 2014).

Dengue is an infection that is present in urban, semi-urban and rural areas. Dengue infection is caused by four serotypes of dengue virus (DV), namely DEN-1, DEN-2, DEN-3 and DEN-4 belonging to genus
Flavivirus and family Flaviviridae. It is spread through the bite of infected Aedes aegypti mosquito. Most primary infections are uneventful. Infection with one serotype confers an individual life-long immunity to that serotype and cross-reactivity to the other serotypes.

The complications like DHF and DSS are usually attributed to this cross-reactivity in secondary infections (Kulkarni et al., 2011).

Mortality from dengue can be reduced by implementing timely, appropriate clinical management, which involves early clinical 
and laboratory diagnosis, intravenous rehydration and other supportive measures (WHO, 2012a).

Laboratory confirmation of dengue infection relies on isolation of the virus in cell culture, the identification of viral nucleic acid or antigens, or the detection of virus specific antibodies (Peeling et al., 2010).

Virus isolation by cell culture is the gold standard test for diagnosis of dengue virus infection (Shu et al., 2004). However, this is not within the reach of peripheral and even most tertiary care laboratories (Peeling et al., 2010; Kulkarni et al., 2011).

Detection of dengue virus-specific $\operatorname{IgM}$ antibodies can be done by the IgM-capture enzyme-linked immunosorbent assay (MACELISA) and/or the rapid dengue immunochromatographic test (ICT). Detection of NS1 has been a promising test for dengue diagnosis in its early febrile stage. The NS1 protein is found to be highly conserved in all dengue serotypes, circulating in high levels during the first few days of illness. New developments in enzyme-linked immunosorbent assay (ELISA) and rapid immunochromographic assays that target nonstructural protein 1 (NS1) have shown high concentrations of this antigen in patients with primary and secondary dengue infections up to 9 days after the onset of illness (Peeling et al., 2010; Datta et al., 2010; Shrivastava et al., 2011).

Thrombocytopenia serves as an indicator to promote the early diagnosis of dengue infection. At times, the platelet count is the only accessory laboratory test available in the peripheral areas that can support the diagnosis of DHF or DSS in clinically suspected cases of dengue infection. Even in remote areas, platelet counts can be roughly estimated by microscopy (WHO, 2011a; Kulkarni et al.,
2011). In this study, correlation of platelet count with dengue parameters was done keeping in mind the constraints in the facilities in the peripheral health system.

\section{Materials and Methods}

The study was conducted in Department of Microbiology, Rajarajeswari medical college and hospital, Bangalore, Karnataka from December 2011 to November 2012 after institutional ethical committee approval. A total of 86 serum samples which were collected from equal number of patients who were clinically suspected of dengue infection (WHO guidelines, 2009) i.e history of fever of sudden onset, with two or more of the following: nausea, vomiting, rash, aches and pains, tourniquet test positive, leukopenia and any warning sign (abdominal pain or tenderness, persistent vomiting, clinical fluid accumulation, mucosal bleed, lethargy, restlessness, liver enlargement > $2 \mathrm{~cm}$, laboratory increase in haematocrit concurrent with rapid decrease in platelet count) and who gave informed consent, were included in the study.

The serum samples were subjected to NS1 ELISA and IgM ELISA using SD Dengue ELISA kits. The tests were performed strictly as per the manufacturer's instructions. Samples with eitherNS1 ELISA positivity or IgM ELISA positivity or both NS1 and IgM ELISA positivity were considered to be dengue positive and the samples which were both NS1 and IgM ELISA negative were considered to be dengue negative. Platelet count of all the patients was noted. Platelet count $\leq 1,00,000$ cells/cubic $\mathrm{mm}$ was considered to be thrombocytopenia.

\section{Statistical analysis}

Chi square test was applied for analysis of categorical data. 


\section{Results and Discussion}

Of the total 86 serum samples tested, 64 samples were tested positive and the remaining 22 samples were negative (Table 1). Of the 64 positive serum samples, 4 (6.25\%) patients were positive for NS1 only, $27(42.19 \%)$ positive for IgM only, while $33(51.56 \%)$ patients were positive for both NS1 and IgM (Table 2). Of the 64 dengue positive patients, thrombocytopenia was noticed in 49 patients $(76.56 \%)$. Of the 4 patients with only NS1 positivity, 2 (50\%) had thrombocytopenia, out of 27 patients with only IgM positivity, 21 (77.78\%) patients had thrombocytopenia and out of 33 patients with both NS1 and IgM positivity, 26 (78.79\%) patients had thrombocytopenia (Table 3). Among the total 64 positive patients, 49 patients had thrombocytopenia and 15 patients had normal platelet count. Among the 22 dengue negative patients, 7 had thrombocytopenia and 15 patients had normal platelet count. On correlation of platelet count with dengue positive and dengue negative parameters by applying Chi-square test, $\mathrm{P}$ value was 0.000145 (that is $\mathrm{P}$ value is $<0.001$ which is highly significant) (Table 4). Hence association between dengue positivity and thrombocytopenia is highly significant.

Chi-square test $\mathrm{P}$ value $=0.000145$

Table 4 shows that thrombocytopenia correlates well with positive dengue parameters

In order to provide timely information for the management of patients, it is important to establish the diagnosis of acute dengue virus infection during the first few days of manifestation of the clinical symptoms (Kumarasamy et al., 2007).

Viral isolation in the cell culture is a timeconsuming process that reproduces viral replication in the host's cells, and for this reason, may not be the appropriate method to be used as the identification routine for acute infections. The PCR techniques demand specific laboratory equipment and suitable physical structure (Dutra et al., 2009).

ELISA and rapid immunochromatographic assays are used for the detection of virus antigen and dengue specific antibodies (Peeling et al., 2010).

The detection of secretory NS1 protein represents a new approach to the diagnosis of acute dengue virus infection. NS1 antigen is detectable as early as day 1 after the onset of fever and is highly specific (Datta et al., 2010; WHO, 2011b; Lapphra et al., 2008).Detection of NS1 antigen by ELISA establishes the diagnosis of dengue virus infection (WHO, 2012b).

The detection of dengue-specific $\operatorname{IgM}$ is a useful diagnostic tool. In primary infections, immunoglobulin $\mathrm{M}$ (IgM) is detected 5 or more days after the onset of illness in the majority of infected individuals. In secondary infections, IgM appears earlier or in the same time frame but are usually at lower titres than in primary infection (Peeling et al., 2010). IgG is a less reliable marker in the diagnosis of DI. Both clinical and sub-clinical infections can produce IgG which may persist for several years affecting the interpretation of test results (Kulkarni et al., 2011; Peeling et al., 2010).

However, in peripheral health system in India, ELISA, PCR, and Viral cell culture cannot be done because of high cost and requirement of technological backup. Sometimes, rapid immunochromatographic test assays also may not be available. Platelet count may become the only accessory laboratory test available, that can provide information which will guide the further management in clinically suspected cases of dengue infection (Kulkarni et al., 2011; WHO, 2011a). 
Table.1 Distribution among clinically suspected cases of dengue infection

\begin{tabular}{|l|c|c|}
\hline Total number of patients & Dengue positive & Dengue negative \\
\hline 86 & 64 & 22 \\
\hline
\end{tabular}

Table.2 Comparison of dengue parameters

\begin{tabular}{|l|c|c|}
\hline Positive parameter & Number of patients & $\%$ \\
\hline NS1 only & 4 & 6.25 \\
\hline IgM only & 27 & 42.1875 \\
\hline NS1 and IgM & 33 & 51.5625 \\
\hline Total & 64 & 100 \\
\hline
\end{tabular}

Table.3 Comparison of platelet count and dengue parameters

\begin{tabular}{|l|c|c|c|}
\hline Positive parameter & $\begin{array}{c}\text { Number of } \\
\text { patients }\end{array}$ & $\begin{array}{c}\text { Platelet count } \leq 1,00,000 \\
\text { cells/cubic } \mathrm{mm}\end{array}$ & $\%$ \\
\hline NS1 only & 4 & 2 & 50 \\
\hline IgM only & 27 & 21 & 77.78 \\
\hline NS1 and IgM & 33 & 26 & 78.79 \\
\hline Total & 64 & 49 & 76.56 \\
\hline
\end{tabular}

Table.4 Correlation of platelet count with dengue parameters

\begin{tabular}{|l|c|c|c|}
\hline \multirow{2}{*}{ Dengue parameter } & Number of patients & \multicolumn{2}{|c|}{ Platelet count } \\
\cline { 3 - 4 } & & Thrombocytopenia & Normal count \\
\hline Positive & 64 & 49 & 15 \\
\hline Negative & 22 & 7 & 15 \\
\hline
\end{tabular}

In a study conducted by Kulkarni et al., using immunochromatography for detection of parameters, the dengue positive parameters correlated well with thrombocytopenia $(P<0.001)$. Of the 320 cases, $220(68.8 \%)$ showed thrombocytopenia. In 150 cases of fever, in which the dengue parameters was negative, thrombocytopenia was noted in $30 \%$ cases (45 of 150) (Kulkarni et al., 2011).

In another similar study conducted by Jyothi et al., association of thrombocytopenia in dengue parameter-positive cases was highly significant $(\mathrm{P}=0.006)$ when compared to thrombocytopenia in dengue parameternegative patients (Jyothi et al., 2015). As per our study, in 64 cases with positive parameter, thrombocytopenia (platelet count $\leq$ $1,00,000 /$ cubic $\mathrm{mm}$ ) was noted in 49 cases (76.6\%) and in 22 cases in which the dengue parameters were negative, thrombocytopenia was noted in 7 cases $(31.8 \%)$.

On applying chi-square test for association, it was found that association of thrombocytopenia and dengue positive parameter was significantly higher compared to thrombocytopenia in dengue negative cases $(P<0.001)$ (Table 4). In our study, NS1 and IgM were detected by Capture ELISA.

Platelet counts are decreased in several other conditions like some viral infections other than dengue, drug induced thrombocytopenia, 
collagen vascular diseases, idiopathic thrombocytopenia etc. (Peters, 2008). However, as seen in above studies and our study, association between thrombocytopenia and dengue positive parameters is more when compared to dengue negative cases.

In this study, correlation of platelet count with dengue parameters was done keeping in mind the constraints in the facilities in the peripheral areas where platelet count may be the only accessory lab test available for dengue infection. In such situations, thrombocytopenia in patients with fever with clinical suspicion of dengue infection, can serve as a predictor of complications of dengue infection.

The limitation of the present study is a small sample size. Also, quantitative ELISA, PCR, Viral cell culture could not be done.

Association of thrombocytopenia with dengue positive parameters being highly significant, if thrombocytopenia is observed in clinically suspected cases of dengue infection, early and prompt therapy should be instituted in order to reduce the morbidity and mortality due to complications of dengue infection.

\section{References}

Datta, S., Wattal, C. 2010. Dengue NS1 antigen detection: A useful tool in early diagnosis of dengue virus infection. Indian J Med Microbiol; 28:107-10.

Dutra, N.R., de Paula, M.B., de Oliveira,M.D., de Oliveira, L.L., de Paula, S.0.2009. The laboratorial diagnosis of dengue: Applications and implications. J Global Infect Dis; 1:3844.

Gupta, E., Ballani, N.2014. Current perspectives on the spread of dengue in India. Infection and Drug Resistance 2014, 7:337-342.
Jyothi, P., Metri, B.C. 2015. Correlation of serological markers and platelet count in the diagnosis of Dengue virus infection. Adv Biomed Res. Jan 30; 4:26. doi: 10.4103/2277-9175.150396.

Kulkarni, R. D., Patil, S. S, Ajantha, G. S., Upadhya, A. K., Kalabhavi, A. S., Shubhada, R.M., Shetty, P. C., Jain, P. A. 2011. Association of platelet count and serological markers of dengue infection- importance of NS1 antigen. Indian J Med Microbiol; 29:359-62.

Kumarasamy, V., Chua, S. K., Hassan, Z., Wahab, A.H.A., Chem, Y.K., Mohamad, M., Chua, K.B. 2007. Evaluating the sensitivity of a commercial dengue NS1 antigen capture ELISA for early diagnosis of acute dengue virus infection. Singapore Med J; 48 (7): 669.

Lapphra, K., Sangcharaswichai, A., Chokephaibulkit, K., Tiengrim, S., Piriyakarnsakul, W. 2008. Evaluation of an NS1 antigen detection for diagnosis of acute dengue infection in patients with acute febrile illness. Diagn Microbiol Infect Dis.; 60:387-391.

Peeling, R.W., Artsob, H., Pelegrino, J.L., Buchy,P., Cardosa, M.J., Devi,S. 2010. Evaluation of diagnostic tests: Dengue. Nat Rev Microbiol; 8: S30-7.

Peters, C.J., 2008. Infections caused by arthropod- and Rodent -borne viruses. In: Fauci, A.S., editor. Harrison's principles of Internal Medicine. 17 th ed. New York: McGraw-Hill Medical Publishing Division; p. 1226-39.

Shrivastava, A., Dash, P. K., Tripathi, N. K., Sahni, A.K., Gopalan, N., Lakshmana Rao, P. V. 2011. Evaluation of a commercial Dengue NS1 enzymelinked immunosorbent assay for early diagnosis of dengue infection. Indian $\mathbf{J}$ Med Microbiol; 29:51-5. 
Shu, P.Y., Huang, J.H.2004. Current advances in dengue diagnosis. Clin Diagn Lab Immunol. 11940:642-650.

WHO, 2009. Dengue guidelines for diagnosis, treatment, prevention and control.p52.

WHO, 2011a. Dengue bulletin vol 35.p75.

WHO, 2011b. Comprehensive guidelines for prevention and control of dengue and dengue hemorrhagic fever. Revised edition. p36.

WHO, 2012a. Global strategy for dengue prevention and control 2012-2020.p3.

WHO, 2012b. Handbook for clinical management of dengue. Geneva.p19.

\section{How to cite this article:}

Kanthishree B. Haritsa, S. Sangeetha and Mamatha B. Patil. 2017. Thrombocytopenia - An Indicator of Impending Complications in Dengue Virus Infection. Int.J.Curr.Microbiol.App.Sci. 6(10): 145-150. doi: https://doi.org/10.20546/ijcmas.2017.610.018 